\title{
Size scaling of the exchange interaction in the quantum Hall effect regime
}

\author{
Daniel Werner (1) and Josef Oswald $\odot^{*}$ \\ Institut für Physik, Montanuniversität Leoben, Franz-Josef-Strasse 18, 8700 Leoben, Austria
}

(Received 3 September 2020; accepted 3 December 2020; published 30 December 2020)

\begin{abstract}
We use the self-consistent Hartree-Fock approximation for numerically addressing the integer quantum Hall effect (IQHE) regime in terms of many-body physics at higher Landau levels (LLs). We investigate the dependence of many-particle interactions on the lateral size of the electron system. We use the exchange enhancement of the $g$-factor for spin-polarized Landau levels as an indicator for the strength of the exchange interaction. The driving force for the $g$-factor enhancement is a Hund's rule behavior for the occupation of spin-split Landau levels that lowers the many-particle ground state energy by arranging as many spins in parallel as possible. By increasing the total number of electrons and total number of available states per LL, it can therefore be expected that the exchange-enhanced spin gap should increase as well. In contrast to the dependence on the magnetic field, an increase of the total number of states by simply increasing the system size at constant magnetic field shows a clear saturation behavior above a lateral system size of $1000 \mathrm{~nm}$. The importance of this result is underlined by an extended introduction, which demonstrates the permanent dominance of many-body interactions in all transport regimes of the IQHE. A modeling of IQHE systems therefore has to include many-body interactions, and our results open a pathway towards many-body modeling of quantum Hall systems of macroscopic size.
\end{abstract}

DOI: 10.1103/PhysRevB.102.235305

\section{INTRODUCTION}

Almost 40 years after its discovery, the quantum Hall effect (QHE) [1] was formally included among the select group of high-precision experiments to form the basis of a new SI system. In his essay to celebrate this achievement [2], von Klitzing also points out "that a microscopic picture of the quantum Hall effect for real devices with electrical contacts and finite current flow is still missing." Looking back over the past 40 years, the QHE has become a major topic at almost every conference dedicated to quantum transport and low-dimensional electron systems at high magnetic fields, even up to the present. Three major research fields can be identified within the integer quantum Hall effect (IQHE) that pinpoint the milestones for theoretical approaches in the past. As research field I, we identify the famous scaling theory that is based on the so-called localization picture of the integer QHE (IQHE). The theoretical basis was composed of narrow quantum channels of noninteracting electrons that are created at the Fermi level in strongly disordered electron systems at high magnetic fields. A major and highly cited paper representing the scaling theory was the review paper of Huckestein [3]. The scaling theory has been successfully justified experimentally and theoretically many times [4-9]. The introduction of an electron-electron (e-e) interaction on the single-particle level (Thomas-Fermi screening, Hartree interactions) opened up research field II. It delivered a more realistic screening

*Josef.Oswald@unileoben.ac.at

Published by the American Physical Society under the terms of the Creative Commons Attribution 4.0 International license. Further distribution of this work must maintain attribution to the author(s) and the published article's title, journal citation, and DOI. behavior of the random bulk potential and the edge potential. The main result was that narrow (edge) channels cannot exist because of the e-e interaction and, instead, the associated screening behavior creates nearly macroscopically wide channels at the Fermi level. Another major and also highly cited representative was the paper of Chklovskii, Shklovskii, and Glazman [10] (CSG). At this point, the wide so-called compressible and incompressible stripes had been established. Those became a real focus of IQHE research and their existence has been confirmed experimentally many times [11-15]. Taking into account many-body interactions finally opened up another research field (III) within the IQHE regime (at this point we exclude the fractional quantum Hall effect from the discussion) that dealt with the spontaneous appearance of density modulations that are shaped as stripes or bubbles in high-mobility samples at larger filling factors. Two papers by Fogler, Koulakov, and Shklovskii (FKS) can be identified as highly cited representatives of this particular research field [16,17] that is still highly active [18-26].

By now, after 40 years of IQHE, one should expect that meanwhile all those mentioned milestones may have grown together in order to work as a well-understood unified picture of the IQHE as a whole. However, looking at the literature delivers a quite unexpected situation: If we associate the above-mentioned three milestones with the also abovementioned highly cited papers and create three groups of papers that are citing them (citing either Huckestein [3], or CSG [10], or FKS [16,17]), we find that each of those groups contains about $450-750$ papers with typically $10 \%$ published in Physical Review Letters (PRL). The reader is encouraged to explore for themselves and find out by comparing those groups of papers that there are almost no common papers in these groups [27]. In other words, there are hardly any papers that cite the Huckestein review and the CSG paper at the same time. The same applies for citations of the FKS 
papers which, at the same time, do not cite the Huckestein and CSG papers. Although there are many hundreds of papers in each group, there are very few papers in more than one group at the same time and only one single paper is in all three of them. That is remarkable, since all these milestones were achieved about 24-28 years ago and by now they together should give a complete and consistent picture of the IQHE. Some sort of synergy between them should be expected to show up as common citations that make use of all three of them. However, this is not the case. This is rather surprising since each group individually contains a huge number of PRL papers citing them, indicating a substantial degree of general importance. However, even though PRL papers usually indicate a broad interest that should go beyond the special topic, we find that here they do not even cross borders between the different research fields within the IQHE. That is an unexpected observation and identifies a critical issue that needs to be resolved. Due to our interpretation, the reason for this is some inconsistency between the theoretical foundations of the scaling theory (research field I) and compressible and incompressible stripes (research field II) on the one hand and the bubble and stripe regime (research field III) on the other hand, as we will show below.

The scaling theory (research field I) is based on narrow channels (channel width of the order of the magnetic length) as obtained for a noninteracting single-particle picture. In contrast, we obtain wide compressible stripes on the basis of interacting single particles, that replace the narrow channels if we "turn on" the e-e interaction according to CSG (research field II). As a consequence, the theory of CSG essentially destroys the theoretical basis for the scaling theory that relies on the existence of narrow quantum channels. However, both theories have been successfully and precisely verified many times for the scaling theory [4-9] as well as for the existence of compressible and incompressible stripes [11-15]. In particular, the scaling of the width of the transition regime between IQHE plateaus is dominated by the transport properties of the bulk region, that, however, becomes a single macroscopically wide compressible stripe within the CSG picture, while it becomes a network of conducting arrow channels according to Chalker and Coddington [28]. The theoretical basis for the scaling theory is a localization/delocalization transition along equipotential lines of the disorder potential, while the states are localized on the length scale of the magnetic length perpendicular to the equipotential lines. If now when considering the theory of CSG the question turns up, what happens to the channel network for quantum percolation? At this point, the theoretical foundations for the scaling theory and the theory of CSG essentially exclude each other [27]. As a consequence, we believe that research fields I and II were developed further by being quite isolated from each other, which is strongly indicated by the missing overlap in the literature. At this point it should be mentioned that it has been attempted to include Thomas-Fermi screening for transport [29]. However, that has been achieved on the basis of the Landauer-Büttiker formalism using transmission and reflection coefficients, and hence localization effects on the basis of quantum percolation as required by the scaling theory have not been applied.

The unification of the theoretical basis for the scaling theory and the CSG picture remained an open question and has been resolved just recently [30,31]. On this basis the picture fundamentally changes in a way that the compressible regions close to half filling exist only on average, and consist of a mixture of clusters of full and empty Laundau levels (LLs), where each type of cluster on average covers half of the area. Only the boundaries of those clusters act as compressible regions and as such perform as narrow channels, and in this way generate a network of quantum channels that exists on top of wide compressible regions [31]. As a consequence, the screening capability is restricted to structure sizes larger than the typical cluster size, such as the wide edge potentials. Therefore the edge potentials can be screened by creating edge potential terraces such as those proposed by CSG, while the fluctuating potential with a smaller mean period remains unscreened and triggers the cluster arrangement. Those in turn create the quantum channel network at their boundaries on top of the potential terrace. In this way the disagreement in the theoretical foundations between research fields I and II is resolved. However, a unifying picture needs to cover also the bubble and stripe regime of clean, high-mobility systems (research field III) as well. This has been achieved recently as we will also outline below.

Moving on to research field III on the background of the FKS papers [16,17], it is obvious that the theoretical approach for these novel stripes and bubbles on the basis of many-body interactions [32] has almost nothing in common with the theoretical approach for the compressible and incompressible stripes introduced by CSG [10] (Thomas-Fermi screening, interacting single-particle picture) as used in the past so far. As a consequence, research field III has also been developed further more or less independently from the other two fields I and II. This fact is again demonstrated by a missing overlap in the citations with the other two research areas [27]. While research field II is based on interacting single particles, research field III is based on many-body interactions. But even if the theoretical approach of fields I, II, and III have nothing in common, they should at least not exclude each other. Furthermore, if increasing the degree of disorder continuously from zero to strong disorder, there are transport regimes of the IQHE where those fields overlap. Consequently, also from the theoretical point of view, compressible and incompressible stripes should be able to coexist with bubbles and stripes as well as they should be able to coexist with narrow channels. With theories that work exclusively in separate regimes and that are based on fundamentally different theoretical foundations, such a task cannot be achieved. In contrast, our approach allows us to study a smooth crossover between these transport regimes from dominating narrow noninteracting channels (field I, scaling theory) to the creation of wide compressible stripes (field II, so far understood on the basis of single-particle interactions) to finally the generation bubbles and stripes (field III, many-body interactions) in low-disorder systems. The latter has been completed very recently [26] and thus completes a unifying picture for all transport regimes of the IQHE [27]. All together we are now able to demonstrate that the discrepancies between the different research fields can only be resolved by taking into account many-body interactions for all these three research fields [26,30,31].

The key mechanism is a Hund's rule behavior for the occupation of the spin-split LLs. The resulting $g$-factor en- 
hancement is then a local quantity depending on the local filling factor. This exchange-enhanced $g$-factor is a concept that allows us to discuss the exchange interaction within the single-electron picture. By doing so, we find that the local variation of the enhancement of the Zeeman energy has to be considered in addition to the laterally varying Hartree potential, leading to a modified effective potential for the electrons that also strongly modifies the screening behavior. In addition to the largely repulsive Hartree part of the self-consistent Thomas-Fermi screening, the dependence on the local filling factor of enhanced Zeeman energy leads to a positive feedback loop in the self-consistent carrier redistribution and produces an instability of the electron density, which may lead to jumps either to a locally full or locally empty LL [26,30,31], resulting in a clustering of the filling factor that is triggered by the disorder or edge potential. The boundaries of those clusters (spin domain walls) finally create narrow channels that align mainly along the edge or random potential fluctuations. As an example, recently, the capability of current transport by spin domain walls has been investigated experimentally in a different material system [33]. In the case of a very clean high-mobility electron system, such a trigger effect for the cluster formation by a random potential is missing and the electron system has to find such a cluster structure by selforganization, resulting in the formation of stripes or bubbles. Before moving on to the main results of this paper, the motivation behind our investigations is outlined briefly below.

The main intention of the above somewhat extended introduction is to convince the reader that we face a permanent presence of many-particle interactions for all transport regimes of the IQHE and any consistent modeling should account for this fact. This makes it necessary to look for concepts to make the Hartree-Fock (HF) method also applicable to electron systems of a macroscopic size, and the present paper should serve as a step towards that direction. If needed to put the whole system into a single Hartree-Fock setup, a modeling of electron systems of macroscopic size seems hopeless since this exceeds any available computing power. Therefore, we investigate how the effect of an exchange interaction scales with sample size. In this context we are going to answer the interesting question as to which extent we lose part of the many-body interactions if dividing a macroscopic system into subsystems addressed by HF individually instead of putting the whole macroscopic system in a single HF setup at once. Below, we will demonstrate that the strength of the exchange interaction saturates with increasing size and not much happens above a size of $1000 \mathrm{~nm}$. On this background it might be sufficient to model a macroscopic electron system by dividing it into plaquettes of $1000 \times 1000 \mathrm{~nm}$ and solving each separately by the HF procedure, and subsequently applying a suitable matching technique for joining them to get the complete system.

\section{METHODS}

\section{A. Self-consistent Hartree-Fock in Landau basis}

In order to model a high-mobility heterostructure in the $\mathrm{QH}$ regime, we consider a two-dimensional electron system (2DES) in an $(x, y)$ plane subject to a perpendicular magnetic field $\vec{B}=B \vec{e}_{z}$ described by the Hamiltonian

$$
\begin{aligned}
H_{2 \mathrm{DES}}^{\sigma}= & h^{\sigma}+V_{\mathrm{C}}=\frac{(\vec{p}-e \vec{A})^{2}}{2 m^{*}}+\frac{\sigma g \mu_{\mathrm{B}} B}{2} \\
& +V_{\mathrm{I}}(\vec{r})+V_{\mathrm{C}}\left(\vec{r}, \vec{r}^{\prime}\right),
\end{aligned}
$$

where $\sigma= \pm \frac{1}{2}$ is a spin degree of freedom, $V_{\mathrm{I}}$ is a smooth random potential modeling the effect of the electron-impurity interaction, $V_{\mathrm{C}}$ represents the electron-electron interaction term, and $m^{*}, g$, and $\mu_{\mathrm{B}}$ are the effective electron mass, $g$-factor, and Bohr magneton, respectively. For the system's many-body state $|\Phi\rangle$, we use the usual ansatz $[34,35]$ of an antisymmetrized product of single-particle wave functions, which we choose as a linear combination of Landau states [36] $\psi_{\alpha}^{\sigma}(\vec{r})=\sum_{n=0}^{N_{\mathrm{LL}}-1} \sum_{k=0}^{N_{\phi}-1} \vec{C}_{n, k}^{\alpha, \sigma} \chi_{n, k}(\vec{r})$, with $N_{\mathrm{LL}}$ being the number of LLs and the periodic Landau functions $\chi_{n, k}(\vec{r})$.

A variational minimization of $\left\langle\Psi\left|H_{2 \mathrm{DES}}\right| \Psi\right\rangle$ with respect to the coefficients $\vec{C}_{n, k}^{\alpha, \sigma}[34,35,37,38]$ yields the self-consistent Hartree-Fock-Roothaan equation [39] $\mathbf{H}^{\sigma} \mathbf{C}^{\sigma}=\mathbf{C}^{\sigma} \mathbf{E}^{\sigma}$, with $\mathbf{C}^{\sigma}=\left(\vec{C}_{1}^{\sigma}, \ldots, \vec{C}_{M}^{\sigma}\right)$ the matrix of eigenvectors and $\mathbf{E}^{\sigma}=$ $\operatorname{diag}\left(\epsilon_{1}^{\sigma}, \ldots, \epsilon_{M}^{\sigma}\right)$ the diagonal matrix of the eigenvalues $\epsilon_{1}^{\sigma} \leqslant$ $\epsilon_{2}^{\sigma} \leqslant \cdots \leqslant \epsilon_{M}^{\sigma}$. Following the Aufbau principle [40], the density matrix is constructed starting from the energetically lowest-lying state up to the Fermi level $\epsilon_{\mathrm{F}}$. In our calculations, we keep $N_{\mathrm{e}}$ fixed and compute $\epsilon_{\mathrm{F}}$ as the energy of the highest occupied state afterwards. We start the self-consistency process using the solution of the noninteracting Hamiltonian $\mathbf{h}^{\sigma}=\left\langle n k\left|h^{\sigma}\right| n^{\prime} k^{\prime}\right\rangle$ as an initial guess for the coefficients $\mathbf{C}^{\sigma}$. From this solution $\mathbf{C}^{(0)}$, we construct the density and Fock matrices and finally the full Hamiltonian [36]. Diagonalization yields an improved solution $\mathbf{C}^{(1)}$. The process continues until convergence of the density matrix has been achieved [36].

\section{B. Data representation}

The data provided by the HF simulation mainly consist of the lateral carrier density distribution. However, the many-particle ground state is composed of an appropriate superposition of different product representations of spin-resolved single-electron states as explained above. Consequently, we also can map out those single-particle states composing the obtained many-particle ground state sorted by spin and ordered by energy. In agreement with the Pauli principle these states appear occupied from the lowest energy up to the energy (Fermi energy) where all available electrons of the system are distributed. If we plot the energy of those single-electron states for each spin separately versus the level index, we get a representation as shown in Fig. 1. The colors are associated with the different spins. In this way it can be nicely seen, that indeed the different spins represent their own level scheme. The bold crosses represent the individual (single-particle) energy levels that are connected by thin lines. The level scheme appears as a dense arrangement creating a bold line at certain energy intervals separated by jumps in energy without any levels (energy gaps) but connected by thin lines for both spin orientations. If looking at the density of the energy levels by projecting all energy levels on the energy axis, we get the more familiar representation in terms of the density of states (DOS) versus energy. Figure 2 shows the spin-resolved plot for the density of states and 


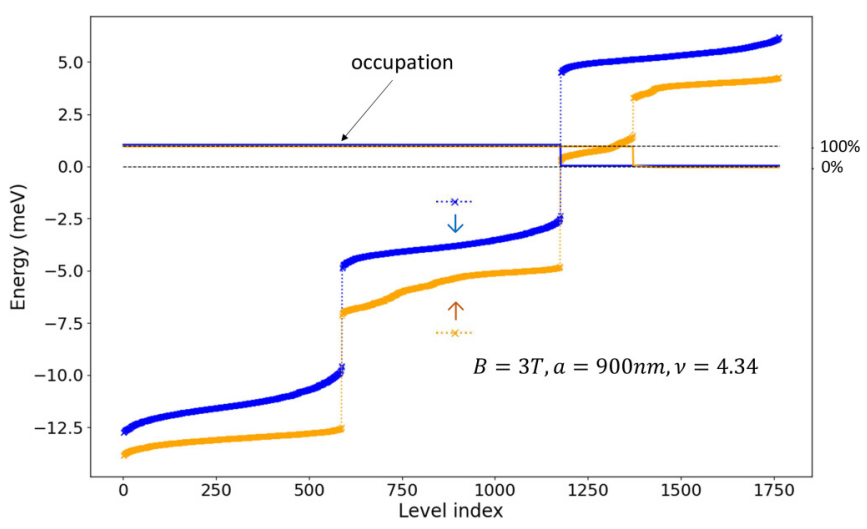

FIG. 1. Energy vs level index for a magnetic field of $3 \mathrm{~T}$, a square specimen side length of $900 \mathrm{~nm}$, and a filling factor of $v=4.34$.

in this way the typical Landau quantization appears. We find well-developed peaks which we can identify as spinresolved LLs separated by energy gaps. However, the common understanding of Landau levels is mostly inspired by the single-electron picture. That means if, e.g., changing the electron density at constant magnetic field, the common, not quite correct, understanding is that the Fermi level just gets pushed through the Landau DOS peaks. Here, we get a large and also qualitative difference that also the Landau peaks tend to move in energy as the Fermi energy changes. As a most striking fact, a gap opens up right at the Fermi level that separates the occupied from the nonoccupied spin-resolved energy levels. This can be nicely seen in Fig. 2 where the orange peak for spin 2 splits up into a peak of occupied and a peak of empty states separated by the green vertical line which represents the Fermi energy. This is mainly due to the exchange-enhanced spin splitting which changes with the filling factor. As an example, if we look for the necessary energy for a spin flip within the topmost partly filled LL, we look for the energy of occupied states of one spin and at the energy of nonoccupied states of the other spin. In Fig. 2 the associated energy dif-

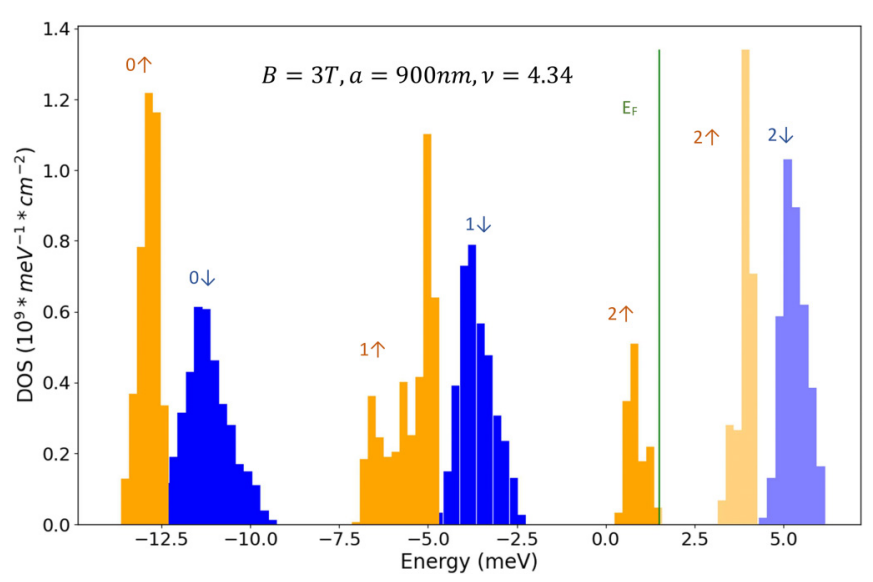

FIG. 2. DOS for a magnetic field of $3 \mathrm{~T}$, a square specimen side length of $900 \mathrm{~nm}$, and a filling factor of $v=4.34$. The vertical green solid line represents the Fermi energy $E_{F}$. The unoccupied states above $E_{F}$ are indicated by a lighter coloring as compared to the occupied states below $E_{F}$.

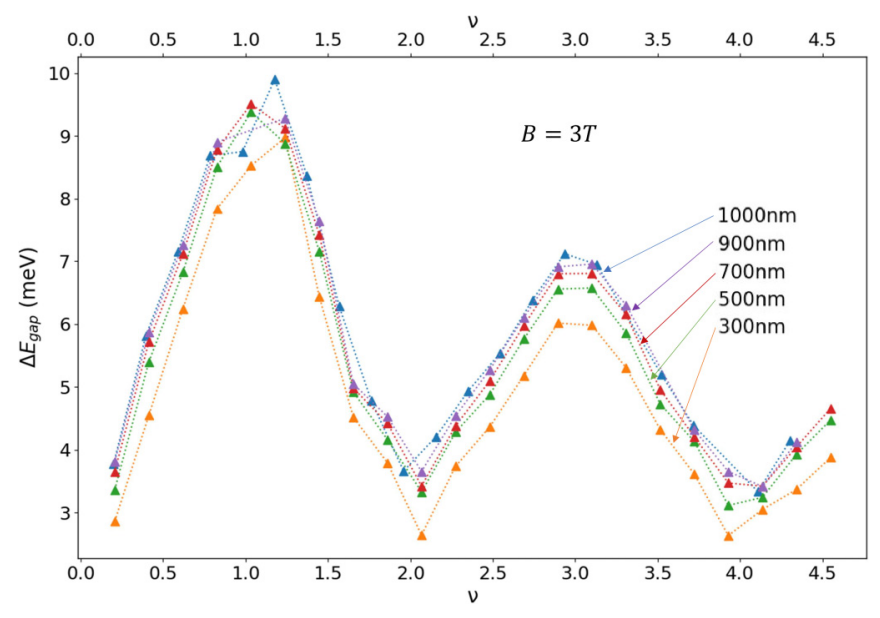

FIG. 3. Exchange-enhanced energy gap $\Delta E_{\text {gap }}$ vs filling factor $v$ for different system sizes at a constant magnetic field of $3 \mathrm{~T}$.

ference for a spin flip is the energy of the occupied orange peak below the Fermi energy (vertical green line) and the energy of the nonoccupied blue peak above the Fermi level. As can be seen, this energy difference is already almost of the order of the LL splitting itself. Experimentally, this appears as a huge Zeemann splitting and is commonly understood as $g$-factor enhancement due to the exchange interaction. Our task in the following is now to extract this exchange-enhanced spin splitting at varying filling factors.

\section{Data evaluation}

In general, the spectrum of spin-split LLs reads as follows,

$$
E=\left(n+\frac{1}{2}\right) \hbar \omega_{C} \pm \frac{1}{2} g^{*} g_{0} \mu_{\mathrm{B}} B
$$

with $n$ the LL index, $\hbar \omega_{C}$ the LL splitting, $\mu_{\mathrm{B}}$ is the Bohr magneton, and $g_{0}$ is the $g$-factor of the free electron. In order to account for the exchange-enhanced spin splitting $g_{0}$ is multiplied by the enhancement factor $g^{*}$. It is likely that the difference in the center of mass on the energy scale of the full and empty DOS peaks of opposite spin in Fig. 2 is a good representation of the spin splitting. However, there are several ways to compute the energy gap. It can be expected that the obtained values may differ slightly depending on the particularly used method of calculation, but the trends important for the purposes of this paper in all of them will be the same. Figure 1 shows the energies of the individual electron states separated for the different spins as discussed above. The rectangle that is built by the occupation functions of the two spins in Fig. 1 defines the interval that contains the states which we use as the candidates for calculating the spin-flip energy. In this range the states indicated by orange stars are occupied, and those represented by blue stars are unoccupied. These will not quite represent the center-of-mass energy of the complete blue peak, but as mentioned already, this might cause a minor shift of the absolute numbers but will not harm the obtained trends. For the calculation of the energy gap $\Delta E_{\text {gap }}$ the average energy difference between those two opposite spin states has been calculated. The enhancement 


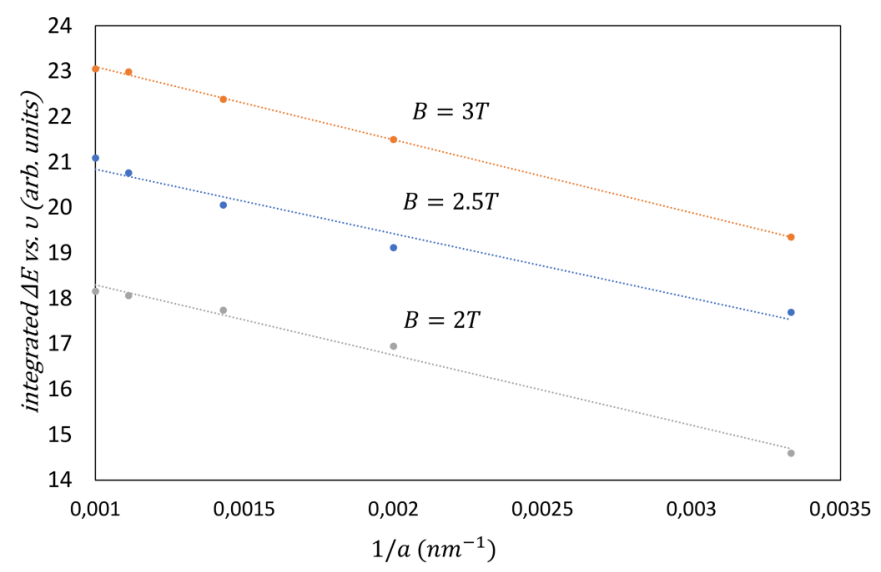

FIG. 4. Integral values of the integrated curves as in Fig. 3 vs inverse specimen square size at different constant magnetic fields. Each data point represents the integral value over a single $\Delta E_{\text {gap }}(v)$ curve.

factor $g^{*}$ can be extracted from the following equation,

$$
\Delta E_{\text {gap }}=g^{*} g_{0} \mu_{\mathrm{B}} B .
$$

At first glance one might have the idea that the most correct way to obtain the spin gap might be the energy difference between the highest occupied (orange) and lowest unoccupied (blue) state. However, one must not forget that the level scheme simply consists of single-particle levels composing the many-particle ground state. A spin flip would change this many-particle state and the lowest unoccupied state will not keep the same energy when becoming occupied. Therefore a one-by-one energy difference between the highest occupied and the lowest unoccupied single-particle states might not be appropriate and not physically correct for defining a gap. In contrast, we do not expect that a single spin flip has a major impact on the average value of all occupied and nonoccupied states of opposite spin. Therefore, it has been chosen to use the whole interval of occupied and unoccupied states within the mentioned rectangle in Fig. 1 for calculating the spin gap.

\section{RESULTS}

The energy gap $\Delta E_{\text {gap }}$ versus filling factor $(\mathrm{FF}) v$ is shown in Fig. 3. Those are the results for different specimen sizes at constant magnetic field as indicated in the figure. As can be observed immediately, an ongoing increase of the length $a$ of the square-shaped sample leads to an ongoing increase of the energy gap but with decreasing progress, which finally manifests in a saturation behavior. As an example, the curves for the 900- and 1000-nm sizes are almost identical already. Instead of looking for the size dependence at a single FF (as, e.g., an odd FF), we get a more statistically reliable quantitative result by using the integral of the whole curves. This has been done by prior fitting splines and subsequent analytical integration. However, in doing so we lose the absolute energy scale for the gap and get arbitrary units instead, but the relative change of the many-body interaction by changing the size remains the same. Looking for a function representing the data, it turns out that we get a linear function if we plot the data against the inverse specimen size $(1 / a)$ as shown in Fig. 4. It suggests that the exchange-driven spin-gap enhancement scales linearly with the inverse system size as $\Delta E_{\text {gap }}=$ $C-D / a$, with $C$ being the saturation value for infinite sample size and $D$ being another constant parameter. The mechanism for this particular linear behavior is not yet clear but such a saturation behavior has to be well expected since the role of many-particle physics should diminish on larger length scales. Looking at Fig. 4, one can estimate that by extrapolating to infinite sample size $(1 / a \rightarrow 0)$ the value should only rise by about $7 \%$ as compared to the value at $a=1000 \mathrm{~nm}$. In other words, the dominating part of the exchange interaction is already captured if restricting the Hartree-Fock procedure to squares of size $1000 \mathrm{~nm}$.

Alternatively, a variation of the magnetic field at fixed sample size shows no saturation behavior. This is finally also the reason why it is possible to express the influence of the exchange interaction by modifying the bare $g$-factor by an enhancement factor $g^{*}$, that appears to be of the order of $g^{*}=15-30$ at odd filling factors. A systematic study of the behavior of $g^{*}$ depending on, e.g., the strength of the disorder is on the way and will be published elsewhere.

\section{DISCUSSION AND OUTLOOK}

The observed linear dependence of the energy gap on the inverse specimen size is so far empiric, but seems well established in the investigated parameter range. A theoretical explanation for the linear function on the inverse system size is still missing and open for a theoretical investigation. However, the main message here is that the data clearly indicate a maximum contribution of the exchange interaction which will not be exceeded if increasing the sample size more and more. This in turn means there exists an upper limit for the size of the electron system which already contains all relevant manybody physics and nothing more is to be expected from HF if modeling still larger system sizes. This is relevant from the practical point of view if aiming at modeling a macroscopicsized specimen. The critical size seems to be $1000 \mathrm{~nm}$ for which it is sufficient to split up a larger electronic system into several pieces of size $1000 \mathrm{~nm}$ for which the HF modeling may be done separately, without losing more than about $7 \%$ of the exchange interaction. This opens up a methodical perspective to extend fully self-consistent laterally resolved HF modeling up to macroscopic system sizes as used experimentally for transport experiments in ultrahigh-mobility QHE samples, by dividing them into appropriate portions and merging them subsequently. A suitable procedure is to be developed in the near future.

\section{ACKNOWLEDGMENTS}

The authors thank Karl Flicker for valuable technical support for the MUL high performance cluster in Leoben, where the Hartree-Fock calculations have been performed. 
[1] K. v. Klitzing, G. Dorda, and M. Pepper, Phys. Rev. Lett. 45, 494 (1980).

[2] K. von Klitzing, Phys. Rev. Lett. 122, 200001 (2019).

[3] B. Huckestein, Rev. Mod. Phys. 67, 357 (1995).

[4] S. Koch, R. J. Haug, K. von Klitzing, and K. Ploog, Phys. Rev. Lett. 67, 883 (1991).

[5] L. W. Engel, D. Shahar, C. Kurdak, and D. C. Tsui, Surf. Sci. 305, 124 (1994).

[6] H. P. Wei, S. Y. Lin, D. C. Tsui, and A. M. M. Pruisken, Phys. Rev. B 45, 3926 (1992).

[7] F. Hohls, U. Zeitler, and R. J. Haug, Phys. Rev. Lett. 88, 036802 (2002).

[8] B. Kramer, T. Ohtsuki, and S. Kettemann, Phys. Rep. 417, 211 (2005).

[9] C. Sohrmann, J. Oswald, and R. Römer, in Quantum and Semi-classical Percolation and Breakdown in Disordered Solids, edited by A. K. Sen, K. K. Bardhan, and B. K. Chakrabarti (Springer, Berlin, 2009), pp. 1-31.

[10] D. B. Chklovskii, B. I. Shklovskii, and L. I. Glazman, Phys. Rev. B 46, 4026 (1992).

[11] A. Siddiki and R. R. Gerhardts, Phys. Rev. B 70, 195335 (2004).

[12] K. Panos, R. R. Gerhardts, J. Weis, and K. von Klitzing, New J. Phys. 16, 113071 (2014).

[13] J. Weis and K. von Klitzing, Philos. Trans. R. Soc., A 369, 3954 (2011).

[14] N. Pascher, C. Rossler, T. Ihn, K. Ensslin, C. Reichl, and W. Wegscheider, Phys. Rev. X 4, 011014 (2014).

[15] E. M. Kendirlik, S. Sirt, S. B. Kalkan, N. Ofek, V. Umansky and A. Siddiki, Nat. Commun. 8, 14082 (2017).

[16] M. M. Fogler, A. A. Koulakov, and B. I. Shklovskii, Phys. Rev. B 54, 1853 (1996).

[17] A. A. Koulakov, M. M. Fogler, and B. I. Shklovskii, Phys. Rev. Lett. 76, 499 (1996).

[18] M. P. Lilly, K. B. Cooper, J. P. Eisenstein, L. N. Pfeiffer, and K. W. West, Phys. Rev. Lett. 82, 394 (1999).

[19] R. R. Du, D. C. Tsui, H. L. Stormer, L. N. Pfeiffer, K. W. Baldwin, and K. W. West, Solid State Commun. 109, 389 (1999).

[20] R. R. Du, W. Pan, H. L. Stormer, D. C. Tsui, L. N. Pfeiffer, K. W. Baldwin, and K. W. West, Physica E 6, 36 (2000).

[21] M. O. Goerbig, P. Lederer, and C. M. Smith, Phys. Rev. B 69, 115327 (2004).
[22] I. V. Kukushkin, V. Umansky, K. von Klitzing, and J. H. Smet, Phys. Rev. Lett. 106, 206804 (2011).

[23] B. Friess, V. Umansky, L. Tiemann, K. von Klitzing, and J. H. Smet, Phys. Rev. Lett. 113, 076803 (2014).

[24] B. Friess, Y. Peng, B. Rosenow, F. von Oppen, V. Umansky, K. von Klitzing, and J. H. Smet, Nat. Phys. 13, 1124 (2017).

[25] B. Friess, V. Umansky, K. von Klitzing, and J. H. Smet, Phys. Rev. Lett. 120, 137603 (2018).

[26] J. Oswald and R. A. Römer, Phys. Rev. B 102, 121305(R) (2020).

[27] See Supplemental Material at http://link.aps.org/supplemental/ 10.1103/PhysRevB.102.235305 for page 1, which outlines the present situation of the three research fields together with the citation statistics that indicates that those research fields appear quite isolated from each other; page 2 , which highlights the problem of incompatible theoretical foundations for research fields I and II; and page 3, which explains that the situation as compared to page 1 drastically changes by providing a unifying theoretical picture if considering many-body interactions dominating all three research fields of the IQHE as demonstrated by our recent work.

[28] J. T. Chalker and P. D. Coddington, J. Phys. C 21, 2665 (1988).

[29] N. R. Cooper and J. T. Chalker, Phys. Rev. B 48, 4530 (1993).

[30] J. Oswald and R. A. Römer, Europhys. Lett. 117, 57009 (2017).

[31] J. Oswald and R. A. Römer, Phys. Rev. B 96, 125128 (2017).

[32] M. M. Fogler, in High Magnetic Fields Applications in Condensed Matter Physics and Spectroscopy, edited by C. Berthier, L. P. Lévy, and G. Martinez, Lecture Notes in Physics Vol. 595 (Springer, Berlin, 2002), p. 98.

[33] A. Kazakov et al., Phys. Rev. Lett. 119, 046803 (2017).

[34] H. Aoki, J. Phys. C 12, 633 (1979).

[35] A. H. MacDonald and G. C. Aers, Phys. Rev. B 34, 2906 (1986).

[36] C. Sohrmann and R. A. Römer, New J. Phys. 9, 97 (2007).

[37] D. Yoshioka and H. Fukuyama, J. Phys. Soc. Jpn. 47, 394 (1979).

[38] A. H. MacDonald and S. M. Girvin, Phys. Rev. B 38, 6295 (1988).

[39] C. C. J. Roothaan, Rev. Mod. Phys. 23, 69 (1951).

[40] E. Cances and C. Le Bris, Int. J. Quantum Chem. 79, 82 (2000). 